\title{
UREMIC STOMATITIS IN CHRONIC RENAL FAILURE
}

Jair C. Leão, Luiz Alcino M. Gueiros, Airton V. Leite Segundo, Alessandra A. T. Carvalho, Willian Barrett, and Stephen R. Porter

Uremic stomatitis is a rarely reported oral mucosal disorder possibly associated with longstanding uremia in chronic renal failure patients. Since it was first mentioned by Lancereaux in 1887 and described by Barié in 1889, there have been only a small number of relevant reports in the literature. Four of 300 patients with uremia were observed to have probable uremic stomatitis in the $1930 \mathrm{~s},{ }^{2}$ while in 1964 another 4 affected patients were reported from a group of 262 patients with renal disease. ${ }^{3}$ The clinical features of uremic stomatitis are poorly defined and are rarely detailed in relevant textbooks. The present report details the clinical and histopathological features of probable uremic stomatitis in a patient with longstanding chronic renal failure and reviews current knowledge of this unusual oral mucosal disorder.

A 46-year-old man was referred by the Nephrology Department of Hospital das Clinicas to the Oral Medicine unit of Universidade Federal de Pernambuco, Recife, Brazil complaining of a burning sensation of the oral mucosa and dysgeusia. The burning sensation affected all oral mucosal surfaces, had been present for the previous 3 months, was continuous, and had no notable initiating or precipitating features. In addition, the patient had had adherent white lesions of the oral mucosa for the previous 3 months that had not resolved with topical nystatin suspension used for 30 days.

Department of Clinic and Preventive Dentistry, Federal University of Pernambuco - Pernambuco/PE, Brazil.

Division of Maxillofacial Diagnostic, Medical and Surgical Sciences, Eastman Dental Institute for Oral Health Care Sciences, UCL, University of London - London, UK.

E-mail: jleao@eastman.ucl.ac.uk
The patient was a taxi driver, married, with 2 healthy children. He had previously drunk up to 20 units of alcohol per week and smoked 40 cigarettes per day, but he had stopped both habits at 22 years of age. He had developed chronic renal disease due to nonspecific nephritis associated with severe hypertension in 1991, at which time the patient commenced hemodialysis. In the same year the patient underwent renal transplantation, but the renal allograft was rejected 4 years later. The patient subsequently developed hepatitis $B$ infection as a consequence of hemodialysis and was treated with interferon for approximately 3 months. He continued hemodialysis 3 times weekly for 10 years until he underwent a further renal transplant in 2001. At the time of his referral to Oral Medicine (late 2003), the patient was on a combination of prednisolone (10 mg daily) and azathioprine (150 mg daily); his renal disease was considered stable aside from some elevation of plasma urea and creatinine. A renal biopsy in 2003 revealed no histopathological features of rejection.

Extra-oral examination revealed no obvious signs of renal disease other than a uremic oral malodor. There was no cervical lymphadenopathy, and the cranial nerves were grossly intact.

Intra-oral examination revealed adherent white plaques of the floor of the mouth, buccal mucosae, lateral borders of the tongue, and gingivae (Figs. 1-3). There were no other mucosal lesions likely to explain the oral mucosal soreness. In view of the extensive nature of the oral lesions, uremic stomatitis was judged to be the working diagnosis; however, the differential diagnosis included oral lichen planus and chronic hyperplastic candidosis. In addition, since the patient had been on immunosuppressive treatment that included azathioprine and prednisolone, oral hairy leucoplakia (OHL) could also account for the clinical signs ob- 


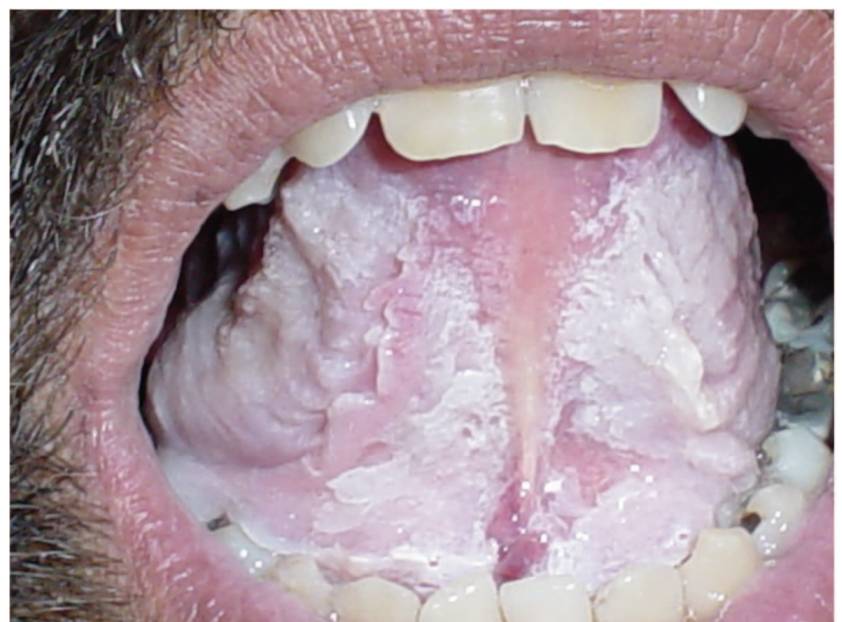

Figure 1 - Adherent white patch on ventral surface of tongue and floor of the mouth.

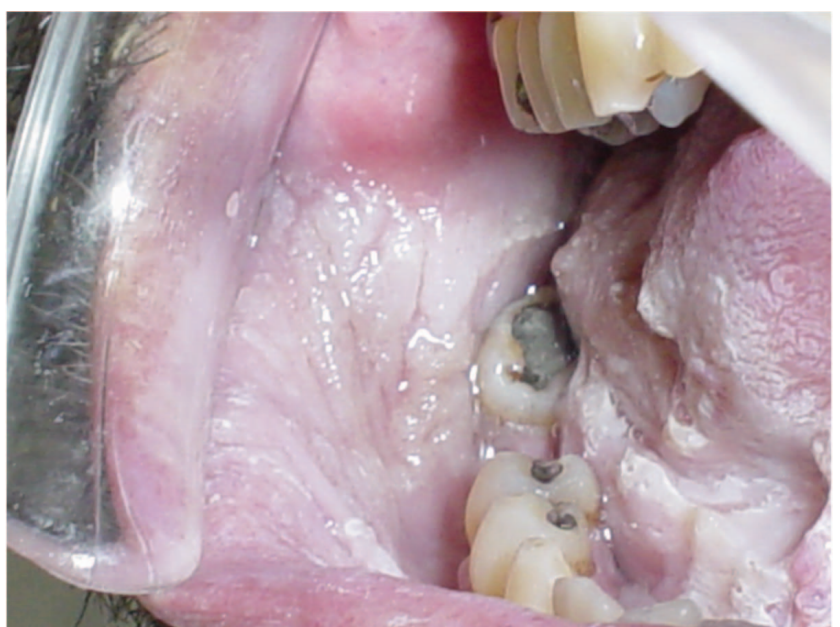

Figure 2 - Widespread white patch on buccal mucosae and floor of the mouth.

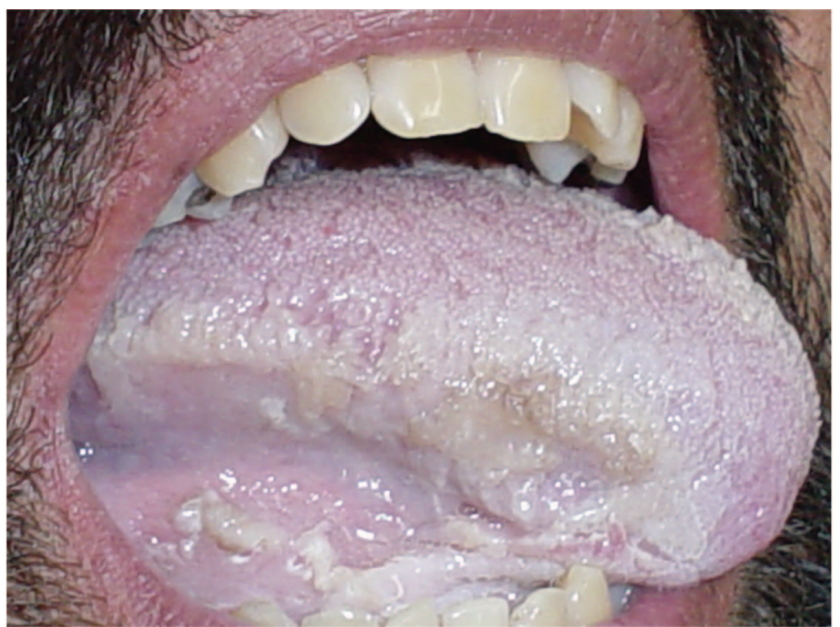

Figure 3 - Adherent white patch on right side of lateral border of tongue. served in this patient. However, OHL seemed unlikely in view of the generalized nature of the oral white patches. To establish the precise diagnosis, incisional biopsies of the left and right borders of the tongue were undertaken.

Histopathogic examination of both biopsy specimens revealed an epithelium that was markedly acanthotic, with most of the suprabasal layers comprising pale-staining degenerate keratinocytes. The surface layers showed sloughing in places, and there was hyperplasia of the basal cell component (Fig. 4). Fungal stains were negative as was immunohistochemistry for human papillomavirus (HPV), Epstein-Barr virus (EBV) latent membrane protein, and EBV early RNA in situ hybridization.

No histological pathognomonic signs of uremic stomatitis have been described. Therefore, based upon the clinical features and lack of histopathological features suggestive of lichen planus, oral hairy leukoplakia, or chronic hyperplastic candidosis, a final diagnosis of uremic stomatitis seemed appropriate. Because the renal disease was generally stable, there was no need to modify the relevant systemic treatment, and a $10 \%$ hydrogen peroxide mouthwash (1:1 in water) 4 times daily was prescribed as a palliative

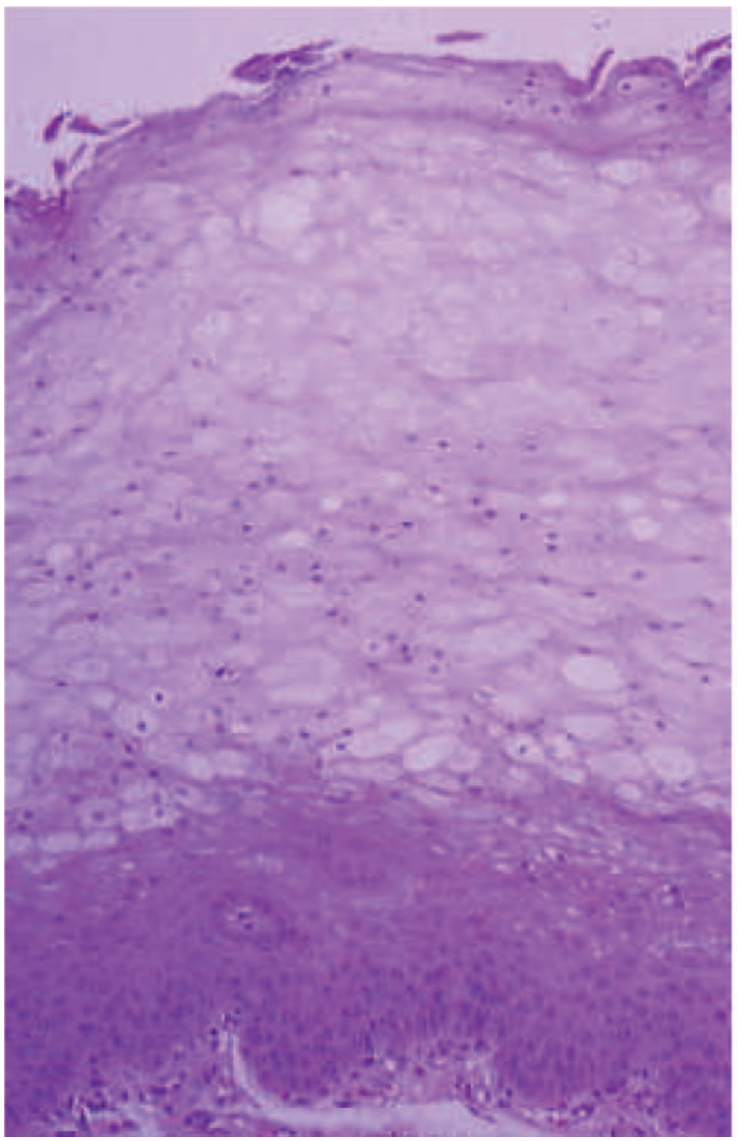

Figure 4 - Histopathologic image of tongue (H\&E), showing an unusual hyperparakeratinization 
therapy. However, perhaps surprisingly, after 2 weeks, the white lesions almost entirely resolved (Figs. 5 and 6) with marked reduction in the burning sensation and taste disturbance. The patient's renal parameters had not changed during this time. The patient was then referred back to the nephrology unit for continued review of his renal disease.

Uremic stomatitis is an uncommon complication of uremia that may occur as a result of advanced renal failure. ${ }^{4-6}$ Since it was first reported, few affected patients have been detailed in the literature.

Affected individuals usually complain of severe oral mucosal discomfort, sometimes interfering with nutrition and fluid intake, reduced salivary flow, dysgeusia, and a burning sensation of the lips.

Uremic stomatitis was first described as a red mucosa covered with a pseudomembrane and ulcerative forms. Clinically, adherent white lesions arise on the dorsal, ven-

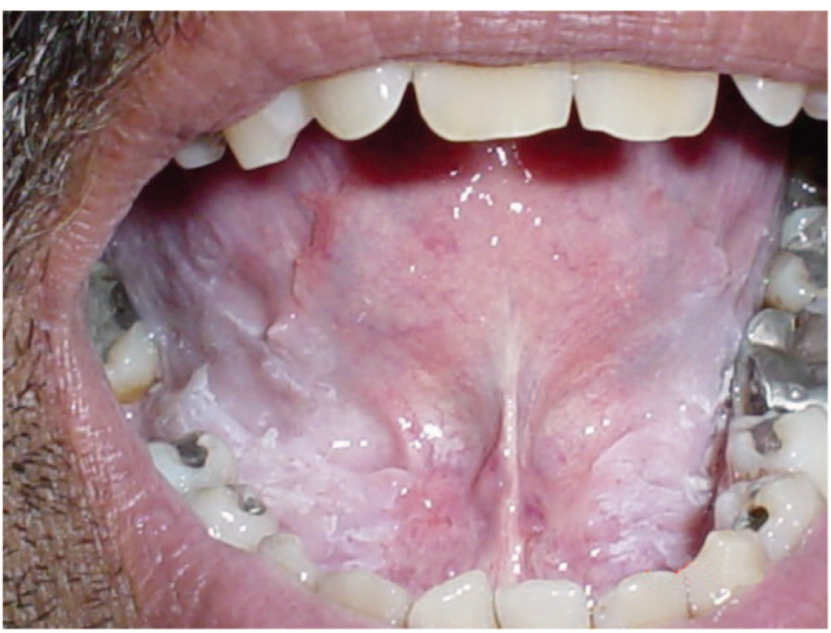

Figure 5 - Ventral surface of tongue and floor of the mouth after 2 weeks of peroxide mouthwash.

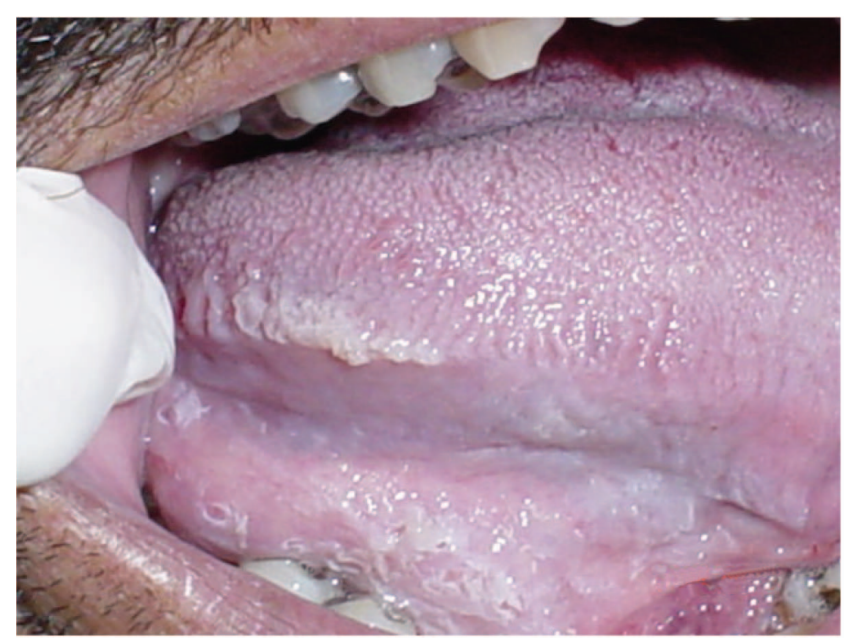

Figure 6 - Lateral border of tongue and floor of the mouth after 2 weeks of peroxide mouthwash. tral, and lateral parts of the tongue, as well as in the buccal, labial, or retro-molar areas. ${ }^{4}$ However, some patients may have an exudate from the oral mucosa, together with ulcerative lesions of the skin. ${ }^{7}$ Gingival and mucosal swelling may also occur. The clinical diagnosis of possible uremic stomatitis is difficult, since the description of the disorder is similar to that of other common diseases affecting the oral mucosa, and few clinical images of the lesions are available.

The etiology of uremic stomatitis remains unknown, although it has been suggested that it may be the consequence of raised levels of ammonia compound. ${ }^{8}$ Uremic stomatitis may also be considered a chemical burning. ${ }^{6} \mathrm{Am}-$ monia is formed through the action of bacterial ureases modifying salivary urea, which can be elevated in affected patients. ${ }^{9}$ It has been suggested that stomatitis appears when blood urea levels are higher than $300 \mathrm{mg} / \mathrm{mL},{ }^{10}$ although, there have been reports of mucosal changes at urea levels of less than $200 \mathrm{mg} / \mathrm{mL}^{8,11}$ In this patient, blood urea levels were $288 \mathrm{mg} / \mathrm{mL}$ (normal range $18-21 \mathrm{mg} / \mathrm{mL}$ ), and unfortunately, this was the only renal function result available.

Other possible causes of mucosal changes associated with uremia include hemorrhagic diathesis, common in uremia, causing decrease of viability of the affected tissues allowing bacterial infection, which can result in ulceration and pseudomembrane formation. ${ }^{4}$

Patients with uremia may have dysgeusia, an altered perception to sweet and sour taste, ${ }^{12}$ and a burning sensation of the lips and tongue possibly caused by pain pathway activation. ${ }^{13}$ Younger patients usually have more significant impairment in taste modalities than older patients, but they may have a better recovery of neural taste function following dialysis. ${ }^{14}$

Zinc deficiency can arise in renal failure ${ }^{15}$ and medically, if zinc levels are very low as in acrodermatitis enteropathica, it could account for mucosal changes. However, there is no evidence that mild zinc deficiency gives rise to oral mucosal changes or oral pain.

Histologically, uremic stomatitis is characterized by minimal underlying inflammatory infiltrate with a hyperplastic epithelium and unusual hyperparakeratinization. ${ }^{7}$

Uremic stomatitis responds to treatment of underlying renal failure. Additionally, local measures aimed at improving oral hygiene may also be used. The present patient had resolution of oral mucosal lesions and symptoms following hydrogen peroxide therapy. This response has been previously observed and perhaps reflects the antimicrobial effect of this agent, thus reducing the local levels of bacterially-derived ureases. ${ }^{5}$

The present report details the clinical and histopatho- 
logical features of uremic stomatitis. The etiology of this oral disorder in unclear; however, as the management of renal disease continues to improve, it is unlikely that many patients will be affected by this rare condition.

\section{ACKNOWLEDGEMENT}

The study was partially funded by a grant from CNPq (Ministry of Science and Technology, Brazil).

\section{REFERENCES}

1. Barie E. De la stomatite uremique. Arch Gen Med. 1889;2:415-32.

2. Hempstead BE, Hench PS. Uremic stomatitis. Proc Staff Meet Mayo Clinic. 1930;5:110-2.

3. Beaney GP. Otolaryngeal problems arising during the management of severe renal failure. J Laryngol Otol. 1964;78:507-15.

4. McCreary CE, Flint SR, McCartan BE et al. Uremic stomatitis mimicking oral hairy leukoplakia: report of a case. Oral Surg Oral Med Oral Pathol Oral Radiol Endod. 1997;83:350-3.

5. Larato DC. Uremic stomatitis: report of a case. J Periodontol. 1975;46: 31-3

6. Halazonetis J, Harley A. Uremic stomatitis. Report of a case. Oral Surg Oral Med Oral Pathol. 1967;23:573-7.

7. Jaspers MT. Unusual oral lesions in a uremic patient. Review of the literature and report of a case. Oral Surg.Oral Med Oral Pathol. 1975;39:934-44.

8. Carlin RT, Seldin R. Oral ulcerations associated with uremia. Report of a case. NY State Dent J. 1969;35:211-4.
9. Bliss S. The cause of sore mouth in nephritis. J Biol Chem. 1937;121:425-7.

10. Gruskin SE, Tolman DE, Wagoner RD. Oral manifestations of uremia. Minn Med. 1970;53:495-9.

11. Bereston ES, Keil H. Membranous stomatitis associated with debilitation and uremia. Arch Dermtatol. 1941;44:562-70.

12. Shepherd R, Farleigh CA, Atkinson C, Pryor JS. Effects of haemodialysis on taste and thirst. Appetite. 1987;9:79-88.

13. Formaker BK, Frank ME. Taste function in patients with oral burning. Chem Senses. 2000;25:575-81.

14. Ciechanover M, Peresecenschi G, Aviram A, Steiner JE. Malrecognition of taste in uremia. Nephron. 1980;26:20-2.

15. Mahajan SK, Abraham J, Migdal SD, Abu-Hamdan DK, McDonald FD. Effect of renal transplantation on zinc metabolism and taste acuity in uremia. A prospective study. Transplantation. 1984;38:599-602. 